\title{
Application of MXCD to Magnetic Thin-Film Sensors
}

\author{
P.J. Bedrossian
}

J.G. Tobin

A.F. Jankowski

G.D. Waddill

T.C. Anthony

J.A. Brug

\section{RECEIVED \\ JUN 241996 \\ OSTI}

This paper was prepared for submittal to the 1996 Spring Meeting of the Materials Research Society San Francisco, CA

April 8-12, 1996

May 17, 1996

This is a preprint of a paper intended for publication in a journal or proceedings. Since changes may be made before publication, this preprint is made available with the understanding that it will not be cited or reproduced without the permission of the author.
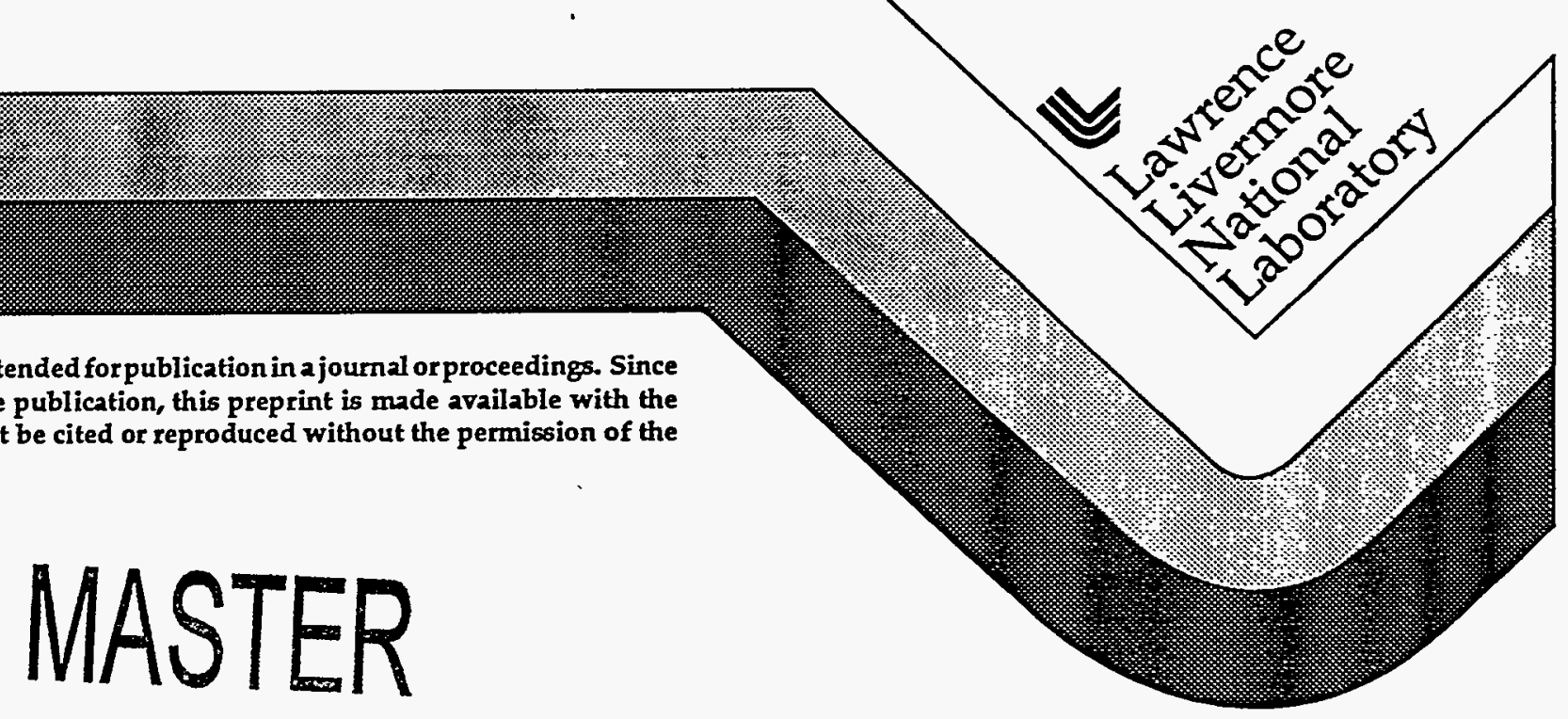

DISTRIBUTION OF THIS DOCUMENT IS UNLLBHTED

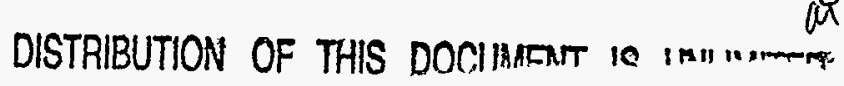




\section{DISCLAIMER}

This document was prepared as an acoount of work sponsored by an agency of the United States Government. Neither the United States Government nor the University of Cahifornia nor any of their employees, makes any warranty, express or implied, or assumes any legal biability or responsibility for the accuracy, completeness, or usefulness of any information, apparatus, product, or process disclosed, or represents that its use would not infringe privately owned rights. Reference herein to any specific commercial product, process, or service by trade name, trademark, manufacturer, or otherwise, does not neossarily constitute or imply its endorsement, recommendation, or favoring by the United States Government or the University of California The views and opinions of authors expressed herein do not necessarily state or reflect those of the United States Government or the University of California, and shall not be used for advertising or product endorsement purposes. 


\title{
APPLICATION OF MXCD TO MAGNETIC THIN-FILM SENSORS
}

\author{
P. J. Bedrossian*, J. G. Tobin*, A. F. Jankowski*, G. D. Waddill**, T. C. Anthony***, \\ and J. A. Brug*** \\ *Lawrence Livermore National Laboratory, Livermore CA 94550 \\ **University of Missouri-Rolla, Rolla MO 65401 \\ ***Hewlett-Packard Laboratories, Palo Alto CA 94305
}

\section{ABSTRACT}

While Magnetic X-ray Circular Dichroism (MXCD) has been applied extensively to the extraction of elemental magnetic moments in various magnetic multilayers, the configuration of actual devices imposes certain constraints on the application of MXCD to devices. Using a set of real, thin-film spin valve devices with varying $\mathrm{Cu}$ spacer layer thicknesses, we demonstrate the correlation between $\mathrm{MXCD}$ and $\mathrm{R}-\mathrm{H}$ measurements on those devices as well as the restrictions on the interpretation of MXCD data imposed by both the device topology and the formulation of realistic error estimates.

\section{INTRODUCTION}

Spin valves, consisting of two, coupled magnetic films separated by a thin ( $<30 \AA ̊)$ spacer layer, offer the oscillatory magnetic coupling of giant magnetoresistance (GMR) devices but with greatly reduced saturation fields as compared with multilayer structures. Because the optimization of spin valve performance depends on precise control of the magnetic moments of coupled layers, the reliable, quantitative measurement of the moments of the constituent elements in the device is critical. The elemental specificity of magnetic circular dichroism ( $M X C D)$ in L-edge $\mathrm{x}$-ray absorption offers the means to detect the magnetic moments of each component of such devices and has already been applied extensively to extract magnetic moments in multilayers. ${ }^{1-4}$ Here we demonstrate the application of $M D X C D$ to actual spin valve devices.

The spin valves consisted of $\mathrm{NiFe}(30 \AA) / \mathrm{Co}(20 \AA) / \mathrm{Cu} / \mathrm{Co}(20 \AA) / \mathrm{NiFe}(50 \AA)$ sandwiches with variable $\mathrm{Cu}$ spacer-layer thickness, grown on a $750 \AA$-thick $\mathrm{NiO}$ film, as described previously. ${ }^{5}$ One permalloy layer is therefore magnerically pinned to the substrate which it contacts. ${ }^{6}$ The magnitude and sign of the coupling of the Iloating layer 
have been shown to oscillate according to the $\mathrm{Cu}$ spacer layer thickness. We present measurements of dichroism in $\mathrm{x}$-ray absorption for three spin valves, with spacer layer thicknesses of $14 \AA, 20 \AA$, and $28 \AA$, which have been associated, respectively, with strong ferromagnetic, antiferromagnetic, and weak ferromagnetic coupling of the floating layer to the pinned layer through the spacer, respectively. The presence of the $50 \AA$-thick outer layer of Permalloy- 80 together with relatively thick $\mathrm{Cu}$ spacer layers in the spin valves, as compared with idealized multilayers used in many previous $\mathrm{MXCD}$ experiments, give the dichroism measurement on the spin valves both elemental and layerwise sensitivity.

\section{EXPERIMENTAL}

Each spin valve was initially magnetized parallel to the sample plane with a 2000 Oersted pulse. Spin-polarized, X-ray absorption spectra were collected according to a procedure described previously, using beamline 8-2 at the Stanford Synchrotron Radiation Laboratory. 2,7 The Poynting vector of the incident beam was aligned at grazing incidence to the films, either parallel or antiparallel to their remanent magnetization. For each sample, four absorption spectra were collected. With the remnant magnetization initially parallel to the incident beam direction, a pair of spectra were recorded, one with the helicity of the incident photons parallel to the remanent magnetization of the device, and one with their helicity antiparallel to the remanent magnetization. Next, the samples were rotated in order to align their remanent magnetization antiparallel to the incident beam direction, and a pair of absorption spectra was similarly obtained from beams polarized with opposite helicity. Finally, each absorption spectrum was normalized to the incident photon flux.

The quantitative extraction of magnetic moments from differences in transition-metal white line intensities arising from $2 p->3 d$ electric dipole transitions has received extensive, theoretical treatment. $3,8-11$ These treatments offer a procedure to extract atomic moments through the insertion of measured white line intensities into sum rules. Although questions remain concerning the absolute accuracy of the sum rules, we will use them for a relative evaluation of atomic moments. The orbital (ORB) and spin moments for each element are then derived from the sum rules (SR) as: 


$$
\mu_{\mathrm{SPIN}}^{\mathrm{SR}}=\frac{6(10-n)\left(\frac{\mathrm{A}^{+}}{\mathrm{C}^{+}}-\frac{\mathrm{A}^{-}}{\mathrm{C}^{-}}\right)}{\left(\frac{\mathrm{A}^{+}}{\mathrm{C}^{+}}+\frac{\mathrm{B}^{+}}{\mathrm{C}^{+}}+\frac{\mathrm{A}^{-}}{\mathrm{C}^{-}}+\frac{\mathrm{B}^{-}}{\mathrm{C}^{-}}\right)}-3\left\langle L_{Z}\right\rangle-7\left\langle T_{Z}\right\rangle
$$

and

$$
\mu_{\mathrm{ORB}}^{\mathrm{SR}}=\left\langle L_{Z}\right\rangle=\frac{4}{3}(10-n) \frac{\left(\frac{\mathrm{A}^{+}}{\mathrm{C}^{+}}+\frac{\mathrm{B}^{+}}{\mathrm{C}^{+}}\right)-\left(\frac{\mathrm{A}^{-}}{\mathrm{C}^{-}} \div \frac{\mathrm{B}^{-}}{\mathrm{C}^{-}}\right)}{\left(\frac{\mathrm{A}^{+}}{\mathrm{C}^{+}}+\frac{\mathrm{B}^{+}}{\mathrm{C}^{+}}\right)+\left(\frac{\mathrm{A}^{-}}{\mathrm{C}^{-}} \div \frac{\mathrm{B}^{-}}{\mathrm{C}^{-}}\right)},
$$

where $n$ is the number of $3 d$ valence electrons, " + " ("-") denotes a spectrum acquired with parallel (antiparallel) helicity and remnant sample magnetization, $A^{i}$ is the integrated intensity of the respective $L_{I I I}$ white line, $\mathrm{B}^{\mathrm{i}}$ the integrated intensity of the respective $L_{I}$ white line, $C^{i}$ equal to the sum of the two underlying continuum step heights, and $T Z$ a magnetic dipole term, which is a small contribution for centro-symmetric systems.

In order to extract the peak intensities from pairs of absorption spectra, we first differentiate the spectra in the vicinity of each elemental $L$-edge peak pair. fit a smooth background on either side of the $L$ peaks, and interpolate through the peak region. Next. we subtract the fit background from the differentiated spectra and integrate the spectra back. The resulting spectra then have the lineshapes of two peaks, each riding on a continuum step. The magnetic moments and associated error estimates are finally extracted from the equations above by considering the full range of peak areas obtained by subtracting out the extreme limits of the possible continuum steps which could be used to fit the spectra.

The layer thicknesses in the spin valves were confirmed with Proton-Induced X-ray Emission (PLXE).

\section{RESULTS AND DISCUSSION}

Figure 1 displays a pair of normalized $\mathrm{MXCD}$ spectra acquired using opposite photon helicities for one of the spin valves. The two spectra acquired with opposite helicity are offset for clarity, and the inset displays the region of the $\mathrm{Cu} L$ lines. The remanent sample magnetization was parallel to the incident $x$-ray beam direction during MXCD measurements. Strong white lines in the absorption spectra are evident at the $L$ - edges of $\mathrm{Fe}, \mathrm{Co}$, and $\mathrm{Ni}$, whereas the small $\mathrm{Cu}$ edge signal from the thin, spacer layer occurs in the region dominated by the Ni EXAFS signal. While the positions and rough 
shapes of the white lines at each absorption edge in fig. 1 are consistent with previous measurements on elemental metals, 12,13 each pair of white lines for a particular element in fig. 1 exhibits a strong dependence of relative intensity on incident beam helicity. The average $\mathrm{Fe}, \mathrm{Co}$, and $\mathrm{Ni}$ magnetic moments in three devices extracted from the sum rules above, together with the results of magnetoresistance measurements, are presented in Table 1. Within the conservative error estimates derived from the procedure described above, the measured spin magnetic moments for $\mathrm{Fe}, \mathrm{Co}$, and $\mathrm{Ni}$ are all less than the saturation moments of $2.21,1.72$, and 0.62 Bohr magnetons, respectively. 14 The details of the magnetotransport measurements have been presented previously. 5

If the measured $x$-ray absorption signal arising from a depth $z$ in the specimen for $a$ given white line energy is proportional to $I(z) \operatorname{Exp}(-z / \mu)$, then the measured, relative intensities of the absorption edges of $\mathrm{Ni}, \mathrm{Fe}$, and $\mathrm{Co}$ in the spectra are consistent with $\mu=22 \AA$. The $C u$ spacer layer, which is buried under at least $13 \AA$ of $C o$ and $50 \AA$ of Permalloy in each specimen, does not contribute appreciably to the absorption signal. Therefore, the MXCD measurement detects not the average magnetic response of different layers but rather the magnetic moment present in the outermost layer for each magnetic element.

For the spin valves with the smallest copper spacer layers, 13 and $15 \AA$, strong ferromagatic coupling between the ferromagnetic layers through the $\mathrm{Cu}$ spacer layer aligns the elemental moments parallel to the remanence, as indicated in Table 1. For the $19 \AA \mathrm{Cu}$ spacer layer, strong anti-ferromagnetic coupling through the $\mathrm{Cu}$ spacer layer forces the moments in the outer $\mathrm{Co}$ and Py layers to align antiparallel to the magnetizing field, as indicated by the negative sign of the extracted moments for that device in Table I. Ferromagnetic coupling atain appears in the two samples with the thickest $\mathrm{Cu}$ spacer layer. For the device with the $21 \AA \mathrm{Cu}$ layer, off-axis rotation of the moments in the outermost Co and Py layers causes near-zero longitudinal moments to be detecred by MXCD.

\section{CONCLUSION}

We have detected and measured the elemental magnetic moments for the ferromagnetic films in a series of real, thin-film, spin valve devices with various $C u$ spacer layer thicknesses. The strong correlation between $\mathrm{MXCD}$ and magnetoresistance measurements on the same devices indicates that the two techniques offer complementar: approaches to the diagnosis of the magnetic microstructure underlying the performance of 


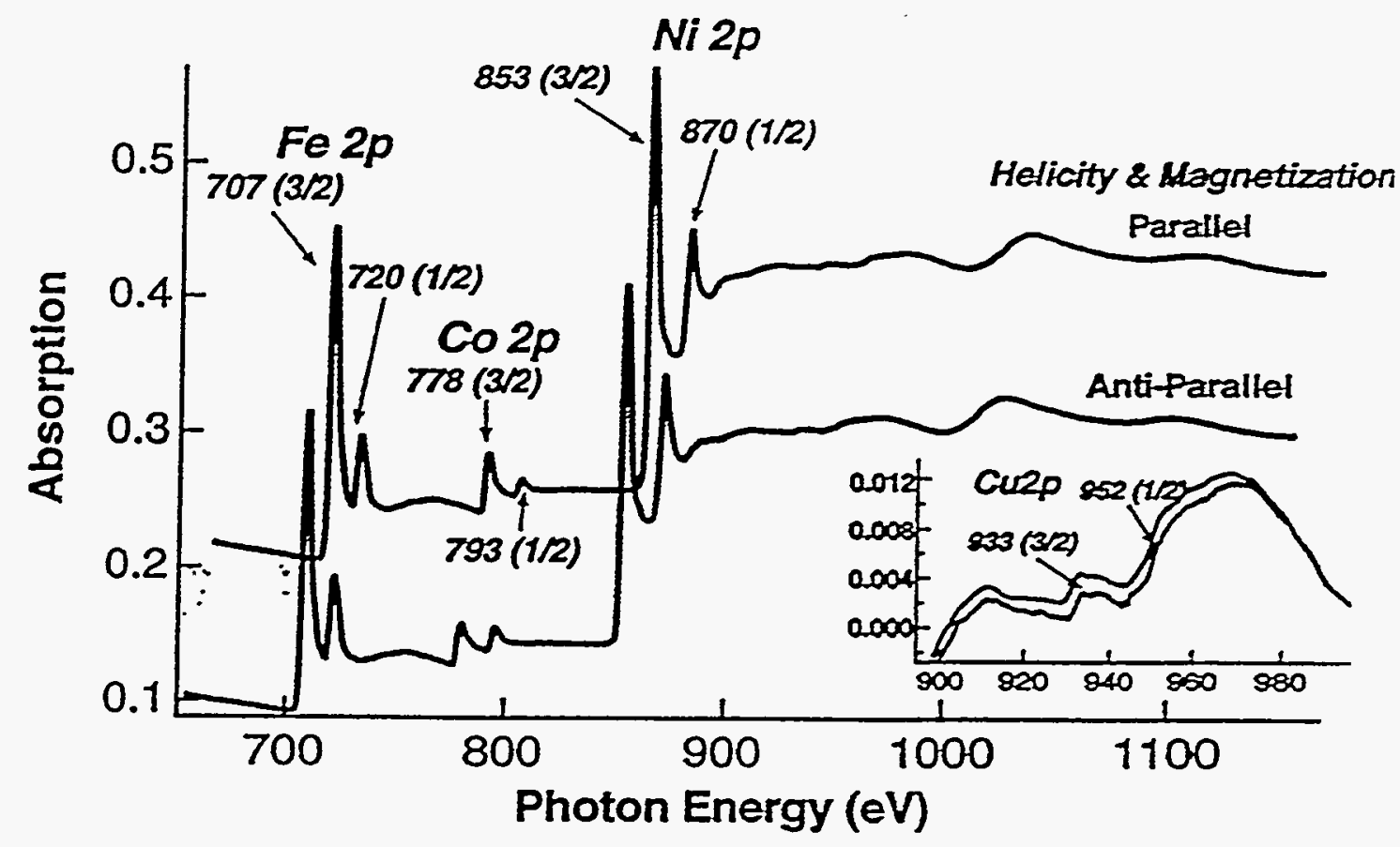

Figure I: Pair of MXCD absorption spectra, acquired with opposite photon helicities, for a spin valve with a $15 \AA \mathrm{Cu}$ spacer layer thickness. The spectrum acquired with the helicity and magnetization aligned parallel is offset in the graph for clarity. The inset shows the detail of the $\mathrm{Cu}$ L-edge.

\section{Elemental Moments from MXCD}

(in Bohr Magnetons)

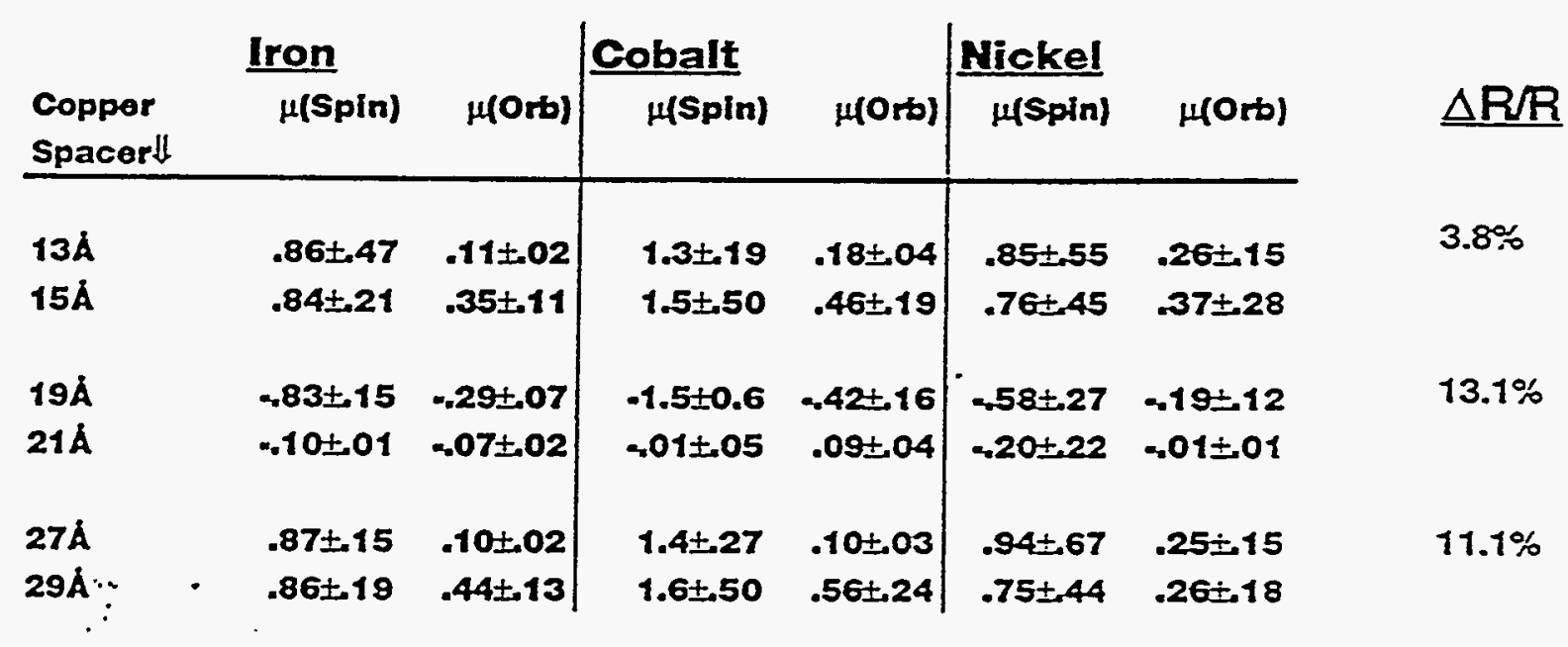

Table I. Elemental magnetic moments for $\mathrm{Fe}, \mathrm{Co}$, and $\mathrm{Ni}$, extracted from 3 IXCD data using the sum rules described in the text, together with the transverse magnetoresistance. 
such devices. While MXCD offers the unique capability to measure elemental moments separately, the application of $\mathrm{MXCD}$ to real thin-film magnetic sensors is subject to restrictions imposed by each device topology.

The authors are grateful to Drs. R. Musket for helpful discussions and G. Bench for PIXE analysis. Research was performed at Lawrence Livermore National Laboratory under the auspices of the US Dept. of Energy under Contract W-7405-Eng-48, and at Hewlett-Packard Laboratories.

\section{REEERENCES}

1. J. Stöhr and Y. Wu. in New Directions in research with 3rd Generation Soft X-ray; Synchrotron Radiation Sources (eds. Schlacter, A. \&.Wuillemier, F.) (Kluwer Academic, Dordrecht; Boston, 1994).

2. A. Jankowski, G. Waddill and J. Tobin. J. Vac. Sci Technol. A 12, 2215-2218 (1994).

3. Y. Wu, et al. Phys. Rev. Lett. 69, 2307-2310 (1992).

4. G. Waddill, J. Tobin and A. Jankowski. J. Appl. Phys. 74, 6999-7001 (1993).

5. T. Anthony, J. Brug and S. Zhang. IEEE Trans. Mag. 30, 3819 (1994).

6. M. Carey and A. Berkowitz. App. Phys. Lett. 60, 3060-3062 (1992).

7. J. Tobin, G. Waddill and D. Pappas. Phys. Rev. Lett. 68, 3642-3645 (1992).

8. J. Erskine and E. Stern. Phys. Rev. B 12, 5016-5024 (1975).

9. N. Smith, C. Chen, F. Sette and L. Mattheiss. Phys. Rev. B 46, 1023-1032 (1992).

10. B. Thole, P. Carra, F. Sette and G.v.d. Laan. Phys. Rev. Lett. 68, 1943-1946 (1992).

11. P. Carra, B. Thole, M. Altarelli and X. Wang. Phys. Rev. Lett. 70, 694-697 (1993).

12. N.K.d. Grande. Physica Scripta 41, 110-114 (1990).

13. R. Leapman, L. Grunes and P. Fejes. Phys. Rev. B 26, 614-635 (1982).

14. C. Chen. Magnetism and Metallury of Soft Magnetic Materials (Dover, New York. 1976). 